\title{
Singular Functions and Characterizations of Field Concentrations: a Survey
}

\author{
Hyeonbae Kang ${ }^{1, *}$ and Sanghyeon $\mathrm{Yu}^{2}$ \\ ${ }^{1}$ Department of Mathematics, Inha University, Incheon 22212, South Korea \\ 2 Department of Mathematics, Korea University, Seoul 02841, South Korea
}

Received 24 August 2020; Accepted (in revised version) 6 October 2020

Dedicated to Prof. Paul H. Rabinowitz with admiration on the occasion of his 80th birthday

\begin{abstract}
In the presence of closely located inclusions of the extreme material property, the physical fields, such as the electric field and the stress tensor, may be concentrated and arbitrarily large in the narrow region between two inclusions. Recently there has been significant progress on the quantitative characterization of the field concentration in the contexts of electrostatics (Laplace equation), linear elasticity (Lamé system), and viscous flow (Stokes system). This paper is to review such progress in a coherent way.
\end{abstract}

Key Words: Field concentration, gradient blow-up, closely spaced inclusions, extreme inclusions, Laplace equation, Lamé system, Stokes system.

AMS Subject Classifications: 35J47, 35B40, 35B45

\section{Introduction}

When two inclusions of the extreme material property are located closely to each other, the physical field may be concentrated and arbitrarily large in the narrow region between the inclusions. An inclusion of the extreme material property means a perfectly conducting or insulating inclusion (the conductivity being $\infty$ or 0 ) in the electrostatic case and a hard inclusion and a hole in the elastostatic case, and the corresponding physical fields are the electric field and the stress tensor. Such field concentration may occur in fiberreinforced composites causing failure of the composites [6], and the electric field can be greatly enhanced and utilized to achieve subwavelength imaging and sensitive spectroscopy [35]. In this respect it is quite important to understand the field concentration in a quantitatively precise manner. It is also quite important to come up with an efficient

*Corresponding author. Email addresses: hbkang@inha.ac.kr (H. Kang), sanghyeon_yu@korea.ac.kr (S. Yu) 
numerical scheme to compute the fields in such cases since numerical computation of the field is known to be very hard in the presence of closely located inclusions.

In response to such importance and mathematical challenges involved in this problem, there has been much progress in understanding the field concentration in the last 20 years or more. In the context of electrostatics (or anti-plane elasticity in two dimensions), the field is the gradient of a solution to the Laplace equation and the precise estimates of the gradient were obtained when the conductivity of the inclusions is $\infty$ : the blow-up rate of the gradient is $\epsilon^{-1 / 2}$ in two dimensions [5,37], where $\epsilon$ is the distance between two inclusions, and it is $|\epsilon \ln \epsilon|^{-1}$ in three dimensions [7]. There is a long list of literature in this direction of research among which we mention $[3,4,9,13,21,27,28,33,34,38]$. We also mention for related works $[10,12,14,22-24]$. If the conductivity of the inclusions is 0 (the insulating case), the two-dimensional problem is dual to the perfectly conducting case (by means of the harmonic conjugation), and hence the blow-up rate of the insulating case is also $\epsilon^{-1 / 2}$. But the three-dimensional case requires further investigation. In this respect, we mention the paper of Yun [39] where a rather unexpected blow-up rate of the gradient has been found when the inclusions are balls. If the conductivity is away from $\infty$ and 0 , then the gradient stays bounded no matter how closely located the inclusions are $[11,30,31]$.

While most of the work mentioned above focus on the estimates from above and below of the blow-up rate of the gradient, there is another important direction of research which is to characterize the singular behavior of the gradient. The characterization of the singular behavior means, roughly speaking, the decomposition of the solution $u$ into the form $u=s+b$ where $s$ carries the information of singularity of the gradient $\nabla u$ and $b$ is a regular function in the sense that $\nabla b$ is bounded (or less singular) regardless of the distance between two inclusions. One important feature of such decompositions is that the singular part $s$ is explicitly given and satisfies the governing equation, e.g., the conductivity equation, the elasticity equation, and so on. It has a significant implication on the numerical computation of the solution in presence of closely located inclusions. Such a computation is known to be a difficult problem because very fine meshes are required since the gradient becomes arbitrarily large in the narrow region. The decomposition enables us to compute the solution $u$ numerically using standard meshes, not refined ones since $s$ is explicit and $b$ is regular. Such a characterization is reminiscent of that related to the corner singularity of elliptic equations which are utilized for computation of the solution to the (interior or exterior) boundary value problem when the domain has a corner $[15,25,26]$.

Characterizations of the field concentration are obtained for the conductivity equation in $[1,16-18,32]$ and for the Lamé system of the linear elasticity in two dimensions in [19] when inclusions are locally strictly convex. These result has been further extended to the two-dimensional Stokes system for circular inclusions [2]. The singular parts of the decomposition are represented by explicit building blocks, which we call singular functions. It is the purpose of this paper to summarize these results on the singularity characterization in a coherent way. 


\section{Geometry of two inclusions}

Let $D_{1}$ and $D_{2}$ be two disjoint simply connected bounded domains in $\mathbb{R}^{2}$ with smooth boundaries. The smoothness assumptions are slightly different depending on the problem, but we assume in this paper that they are $C^{\infty}$ smooth just for simplicity. The domains $D_{1}$ and $D_{2}$ are inclusions. Let $D^{e}$ be the outside of the inclusions, namely, $D^{e}:=$ $\mathbb{R}^{2} \backslash \overline{D_{1} \cup D_{2}}$, and let

$$
\epsilon:=\operatorname{dist}\left(D_{1}, D_{2}\right) \text {. }
$$

Suppose that there are unique points $\mathbf{z}_{1} \in \partial D_{1}$ and $\mathbf{z}_{2} \in \partial D_{2}$ such that

$$
\left|\mathbf{z}_{1}-\mathbf{z}_{2}\right|=\operatorname{dist}\left(D_{1}, D_{2}\right) .
$$

We assume that $D_{j}$ is strictly convex near $\mathbf{z}_{j}$, namely, there is a common neighborhood $U$ of $\mathbf{z}_{1}$ and $\mathbf{z}_{2}$ such that $D_{j} \cap U$ is strictly convex for $j=1,2$. Moreover, we assume that

$$
\operatorname{dist}\left(D_{1}, D_{2} \backslash U\right) \geq C \text { and } \operatorname{dist}\left(D_{2}, D_{1} \backslash U\right) \geq C
$$

for some positive constant $C$ independent of $\epsilon$. This assumption says that points in $D_{1}$ and $D_{2}$ other than neighborhoods of $\mathbf{z}_{1}$ and $\mathbf{z}_{2}$ are at some distance to each other. Note that strictly convex domains satisfy all the assumptions.

Let $\kappa_{j}$ be the curvature of $\partial D_{j}$ at $\mathbf{z}_{j}$. Let $B_{j}$ be the disk osculating to $D_{j}$ at $\mathbf{z}_{j}(j=1,2)$. Then the radius $r_{j}$ of $B_{j}$ is given by $r_{j}=1 / \kappa_{j}$. Let $R_{j}$ be the inversion with respect to $\partial B_{j}$ and let $\mathbf{p}_{1}$ and $\mathbf{p}_{2}$ be the unique fixed points of the combined reflections $R_{1} \circ R_{2}$ and $R_{2} \circ R_{1}$, respectively. We emphasize that $\partial B_{1}$ and $\partial B_{2}$ are circles of Apollonius of $\mathbf{p}_{1}$ and $\mathbf{p}_{2}$.

After rotation and translation, we assume that $\mathbf{p}=\left(\mathbf{p}_{1}+\mathbf{p}_{2}\right) / 2$ is at the origin and the $x$-axis is parallel to the vector $\mathbf{p}_{2}-\mathbf{p}_{1}$. Then one can see (cf. [5]) that $\mathbf{p}_{1}$ and $\mathbf{p}_{2}$ are written as

$$
\mathbf{p}_{1}=(-a, 0) \text { and } \mathbf{p}_{2}=(a, 0),
$$

where the constant $a$ satisfies

$$
a=\sqrt{\frac{2}{\kappa_{1}+\kappa_{2}}} \sqrt{\epsilon}+\mathcal{O}\left(\epsilon^{3 / 2}\right) .
$$

The geometry of $D_{1}$ and $D_{2}$ is depicted in Fig. 1 which is taken from [19].

\section{Conductivity equation}

The conductivity problem with two conducting inclusions is modelled as follows:

$$
\begin{cases}\Delta u=0 & \text { in } D^{e}, \\ u=\lambda_{j}(\text { constant }) & \text { on } \partial D_{j}, \quad j=1,2 \\ u(\mathbf{x})-h(\mathbf{x})=\mathcal{O}\left(|\mathbf{x}|^{-1}\right) & \text { as }|\mathbf{x}| \rightarrow \infty\end{cases}
$$




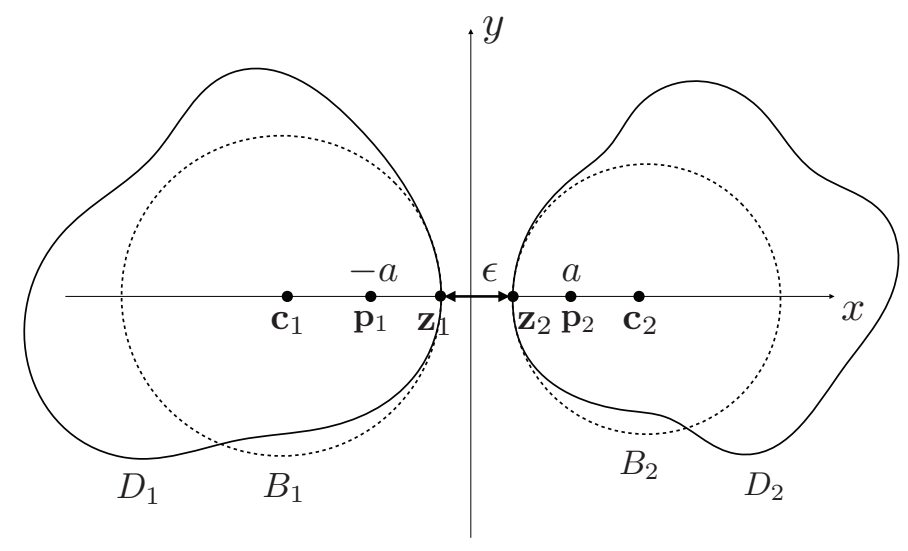

Figure 1: Geometry of the two inclusions and osculating circles [19].

where $h$ is a given function harmonic in $\mathbb{R}^{2}$. The fact that the solution $u$ takes a constant value on $\partial D_{j}$ indicates that $D_{j}$ is a perfect conductor meaning that its conductivity is $\infty$. The constants $\lambda_{j}$ are not prescribed and the problem (3.1) is not an exterior Dirichlet problem. The constants are rather determined by the conditions

$$
\int_{\partial D_{j}} \nabla u \cdot \mathbf{n} d \sigma=0, \quad j=1,2
$$

Here and throughout this paper, $\mathbf{n}$ denotes the outward normal on $\partial D_{j}(j=1,2)$. It is worth emphasizing that the constants $\lambda_{1}$ and $\lambda_{2}$ may or may not be the same depending on the given $h$; When they are different, there occurs a sharp gradient if the distance between $D_{1}$ and $D_{2}$ is short.

The singular function for the conductivity problem is given by

$$
q(\mathbf{x})=\frac{1}{2 \pi}\left(\ln \left|\mathbf{x}-\mathbf{p}_{1}\right|-\ln \left|\mathbf{x}-\mathbf{p}_{2}\right|\right),
$$

where $\mathbf{p}_{j}(j=1,2)$ are the fixed points of the combined reflections as defined in section 2 . The function $q$ has important properties including the following:

(i) It is harmonic in $\mathbb{R}^{2}$ except at $\mathbf{p}_{1}$ and $\mathbf{p}_{2}$.

(ii) It takes constant values on osculating circles $\partial B_{j}$. It is because $\partial B_{1}$ and $\partial B_{2}$ are circles of Apollonius of $\mathbf{p}_{1}$ and $\mathbf{p}_{2}$.

(ii) It holds that

$$
\int_{\partial D_{1}} \nabla q \cdot \mathbf{n} d \sigma=-1, \quad \int_{\partial D_{2}} \nabla q \cdot \mathbf{n} d \sigma=1
$$


This function appears in the bipolar coordinate system for $\partial B_{1}$ and $\partial B_{2}$ as will be seen in section 5. It was used for analysis of the field concentration for the first time in [37].

The singular behaviour of the solution $u$ to (3.1) can be characterized in terms of the function $q$. In fact, the following decomposition formula is obtained in [16], which is an improvement upon the corresponding decomposition in [1]:

$$
u=C_{0} q+b
$$

where $C_{0}$ is a constant independent of $\epsilon$ and $b$ is a function which depends on $\epsilon$ ( $q$ also depends on $\epsilon$ ), but whose gradient is bounded on any bounded subset of $D^{e}$. To explain what the constant $C_{0}$ is, let $D_{j}^{0}(j=1,2)$ be the domain obtained from $D_{j}$ by the translation which moves the point $\mathbf{z}_{j}$ to the origin. The domains $D_{1}^{0}$ and $D_{2}^{0}$ are touching to each other at the origin. Let $u_{0}$ be the solution to (3.1) with $D_{j}$ replaced with $D_{j}^{0}, j=1,2$, namely, the solution for the touching case. In this case, the constant values of $\partial D_{j}^{0}$ are the same and $u_{0}$ is regular even if the exterior domain has cusps. The constant $C_{0}$ is given by

$$
C_{0}:=\int_{\partial D_{1}^{0}} \nabla u_{0} \cdot \mathbf{n} d \sigma
$$

A way to compute $C_{0}$ numerically is proposed in [16].

The decomposition formula (3.5) has some important consequences. Since $\nabla q$ is bounded from below and above by $\epsilon^{-1 / 2}$ (up to constant multiples), the blow-up estimates for $\nabla u$ can be obtained from (3.5). As mentioned before, it can be used to compute $u$ numerically. Since (3.5) extracts the major singular term in an explicit way, it suffices to compute the residual term $b$ for which only regular meshes are required. This idea appeared and was exploited in [17] in the special case when $D_{j}$ 's are disks. There the decomposition formula was derived when $D_{j}$ 's are disks (of the radius $r_{j}$ ) with the constant $C_{0}$ replace with

$$
\frac{4 \pi r_{1} r_{2}}{r_{1}+r_{2}} \frac{\left(\mathbf{z}_{2}-\mathbf{z}_{1}\right) \cdot \nabla h(\mathbf{p})}{\left|\mathbf{z}_{2}-\mathbf{z}_{1}\right|}
$$

The formula (3.5) has another very interesting implication. The quantity $\nabla u \cdot \mathbf{n}$ represents the charge density on $\partial D_{1} \cup \partial D_{2}$ induced by the field $-\nabla h$, and $\nabla u_{0} \cdot \mathbf{n}$ does that on $\partial D_{1}^{0} \cup \partial D_{2}^{0}$. Note that the charge densities on the separating inclusions have a singular part $C_{0} \nabla q \cdot \mathbf{n}$ and a regular part $\nabla b \cdot \mathbf{n}$. It is proved in [16] that $\nabla b \cdot \mathbf{n}$ converges to $\nabla u_{0} \cdot \mathbf{n}$ as $\epsilon \rightarrow 0$, or as the separating inclusions approach to the touching ones. So the singular part suddenly disappears when two inclusions become touching. It is reminiscent of the electrical spark between two separated conductors which suddenly disappears when the conductors are touching.

The decomposition formula of the kind (3.5) when $D_{1}$ and $D_{2}$ are three-dimensional balls of the same radii has been derived in [18] (see [29] for the case of different radii). In this case the singular function is given as an infinite sum of point charges. 


\section{Lamé system}

To describe the elasticity problem with two hard inclusions, let $(\lambda, \mu)$ be the pair of Lamé constants of $D^{e}$ which satisfies the strong ellipticity conditions: $\mu>0$ and $\lambda+\mu>0$ (we only consider the two-dimensional case). Then the problem is given as follows in terms of the Lamé system of equations

$$
\mathcal{L}_{\lambda, \mu} \mathbf{u}:=\mu \Delta \mathbf{u}+(\lambda+\mu) \nabla \nabla \cdot \mathbf{u}
$$

here, $\mathbf{u}=\left(u_{1}, u_{2}\right)^{T}$ is a vector-valued function:

$$
\begin{cases}\mathcal{L}_{\lambda, \mu} \mathbf{u}=0 & \text { in } D^{e}, \\ \mathbf{u}=\sum_{j=1}^{3} c_{i j} \Psi_{j} & \text { on } \quad \partial D_{i}, \quad i=1,2 \\ \mathbf{u}(\mathbf{x})-\mathbf{H}(\mathbf{x})=\mathcal{O}\left(|\mathbf{x}|^{-1}\right) & \text { as }|\mathbf{x}| \rightarrow \infty\end{cases}
$$

where $\mathbf{H}=\left(h_{1}, h_{2}\right)^{T}$ is a given function satisfying $\mathcal{L}_{\lambda, \mu} \mathbf{H}=0$ in $\mathbb{R}^{2}$. Here, $\Psi_{j}$ are the displacement fields of the rigid motions defined by

$$
\Psi_{1}(\mathbf{x})=\left[\begin{array}{l}
1 \\
0
\end{array}\right], \quad \Psi_{2}(\mathbf{x})=\left[\begin{array}{l}
0 \\
1
\end{array}\right], \quad \Psi_{3}(\mathbf{x})=\left[\begin{array}{c}
-y \\
x
\end{array}\right]
$$

The boundary conditions to be satisfied by the displacement $\mathbf{u}$ on $\partial D_{j}$ (the second line in (4.1)) indicate that $D_{1}$ and $D_{2}$ are hard inclusions, and the constants $c_{i j}$ are not given but determined by the conditions

$$
\int_{\partial D_{i}} \Psi_{j} \cdot \sigma[\mathbf{u}] \mathbf{n} d \sigma=0, \quad i=1,2, \quad j=1,2,3 .
$$

Here $\sigma[\mathbf{u}]$ denotes the stress tensor corresponding to the displacement vector $\mathbf{u}$ defined by

$$
\sigma[\mathbf{u}]:=\lambda(\nabla \cdot \mathbf{u})+2 \mu(\widehat{\nabla} \mathbf{u}),
$$

where $\hat{\nabla} \mathbf{u}=\frac{1}{2}\left(\nabla \mathbf{u}+\nabla \mathbf{u}^{T}\right)$ ( $T$ for transpose).

To present singular function for the elasticity problem, let $\boldsymbol{\Gamma}=\left(\Gamma_{i j}\right)_{i, j=1}^{2}$ be the Kelvin matrix of fundamental solutions to the Lamé operator $\mathcal{L}_{\lambda, \mu}$, namely,

$$
\Gamma_{i j}(\mathbf{x})=\frac{\alpha_{1}}{2 \pi} \delta_{i j} \ln |\mathbf{x}|-\frac{\alpha_{2}}{2 \pi} \frac{x_{i} x_{j}}{|\mathbf{x}|^{2}}
$$

where

$$
\alpha_{1}=\frac{1}{2}\left(\frac{1}{\mu}+\frac{1}{\lambda+2 \mu}\right) \quad \text { and } \quad \alpha_{2}=\frac{1}{2}\left(\frac{1}{\mu}-\frac{1}{\lambda+2 \mu}\right)
$$


The singular functions for the problem (4.1) are obtained in [19] as linear combinations of point source functions in linear elasticity called nuclei of strain. The following nuclei of strain are used

$$
\boldsymbol{\Gamma}(\mathbf{x}) \mathbf{e}_{1}, \quad \boldsymbol{\Gamma}(\mathbf{x}) \mathbf{e}_{2}, \quad \frac{\mathbf{x}}{|\mathbf{x}|^{2}}, \quad \frac{\mathbf{x}^{\perp}}{|\mathbf{x}|^{2}}
$$

where

$$
\mathbf{e}_{1}=(1,0)^{T}, \quad \mathbf{e}_{2}=(0,1)^{T} \quad \text { and } \quad(x, y)^{\perp}=(-y, x) .
$$

It turns out that those singular functions can be expressed in the simple forms using the function $\zeta$ :

$$
\begin{aligned}
& \mathbf{q}_{1}=\alpha_{1}\left[\begin{array}{l}
q \\
0
\end{array}\right]-\alpha_{2} x_{1} \nabla q \\
& \mathbf{q}_{2}=\alpha_{1}\left[\begin{array}{l}
0 \\
q
\end{array}\right]+\alpha_{2} x_{1}(\nabla q)^{\perp}
\end{aligned}
$$

where $q$ is the singular function for the conductivity problem given in (3.3). One can easily see that $\mathbf{q}_{j}$ are solutions to the Lamé system, namely,

$$
\mathcal{L}_{\mu, \lambda} \mathbf{q}_{j}=0 \text { in } \mathbb{R}^{2} \backslash\left\{\mathbf{p}_{1}, \mathbf{p}_{2}\right\}
$$

It is shown in [19] that $\mathbf{q}_{j}$ takes 'almost' constant values on the osculating circles $\partial B_{i}$ $(i=1,2)$. In fact, there are constant $\alpha_{i j}$ and $\beta_{i j}$ (which depends on $\epsilon$ ) such that

$$
\mathbf{q}_{j}(\mathbf{x})=\alpha_{i j} \Psi_{j}(\mathbf{x})+\beta_{i j} \mathbf{x}, \quad \mathbf{x} \in \partial B_{i}, \quad i, j=1,2 .
$$

Another function related with the boundary value $\Psi_{3}$ on $\partial B_{1}$ and $\partial B_{2}$ is constructed in the same paper. But this function has nothing to do with the singular behavior of the field, so we omit it here. It is worth mentioning that the singular functions $\mathbf{q}_{1}$ and $\mathbf{q}_{2}$ are effectively utilized to prove the Flaherty-Keller formula on the effective property of densely packed elastic composites [20].

Using the singular functions $\mathbf{q}_{1}$ and $\mathbf{q}_{2}$, it is proved that the solution $\mathbf{u}$ to (4.1) admits the following decomposition:

$$
\mathbf{u}=C_{1} \mathbf{q}_{1}+C_{2} \mathbf{q}_{2}+\mathbf{b},
$$

where $C_{1}$ and $C_{2}$ are constants depending on $\epsilon$, but bounded independently of $\epsilon$, and $\mathbf{b}$ is a function whose gradient is bounded on any bounded subset of $D^{e}$. This decomposition formula enables us to prove that $\epsilon^{-1 / 2}$ is an upper bound of $\nabla \mathbf{u}$, and it is also a lower bound in some cases. The fact that $\epsilon^{-1 / 2}$ is an upper bound of $\nabla \mathbf{u}$ was proved in [8].

We mention that the constants $C_{1}$ and $C_{2}$ appearing in the formula (4.10) are not explicit. Thus further investigation on how to determine them (or compute them numerically) is desired. 


\section{Stokes system}

We also consider the Stokes system in the exterior domain $D^{e}$. Let $(\mathbf{U}, P)$ is a given background solution to the homogeneous Stokes system in $\mathbb{R}^{2}$, namely,

$$
\mu \Delta \mathbf{U}=\nabla P \text { in } \mathbb{R}^{2} .
$$

We consider the following problem of the Stokes system:

$$
\begin{cases}\mu \Delta \mathbf{u}=\nabla p & \text { in } D^{e}, \\ \nabla \cdot \mathbf{u}=0 & \text { in } D^{e}, \\ \mathbf{u}=\sum_{j=1}^{3} d_{i j} \Psi_{j} & \text { on } \partial D_{i}, \quad i=1,2\end{cases}
$$

with the conditions

$$
(\mathbf{u}-\mathbf{U})(\mathbf{x})=\mathcal{O}\left(|\mathbf{x}|^{-1}\right), \quad \nabla(\mathbf{u}-\mathbf{U})(\mathbf{x})=\mathcal{O}\left(|\mathbf{x}|^{-2}\right), \quad(p-P)(\mathbf{x})=\mathcal{O}\left(|\mathbf{x}|^{-2}\right),
$$

as $|\mathbf{x}| \rightarrow \infty$. Here $\mu$ represents the constant viscosity of the fluid, $\Psi_{j}$ are the functions given in (4.2), and $d_{i j}$ are constants to be determined from the equilibrium conditions

$$
\int_{\partial D_{i}} \Psi_{j} \cdot \sigma[\mathbf{u}, p] \mathbf{n} d \sigma=0, \quad i=1,2, \quad j=1,2,3 .
$$

Here, $\sigma[\mathbf{u}, p]$ is the stress field induced by the velocity-pressure pair $(\mathbf{u}, p)$, namely,

$$
\sigma[\mathbf{u}, p]=-p I+2 \mu \widehat{\nabla} \mathbf{u},
$$

where $I$ is the identity matrix.

The singular functions $\left(\mathbf{h}_{j}, p_{j}\right), j=1,2$, for the problem (5.2) is the solution to the following problem:

$$
\begin{cases}\mu \Delta \mathbf{h}_{j}=\nabla p_{j} & \text { in } \mathbb{R}^{2} \backslash\left\{\mathbf{p}_{1}, \mathbf{p}_{2}\right\}, \\ \nabla \cdot \mathbf{h}_{j}=0 & \text { in } \mathbb{R}^{2} \backslash\left\{\mathbf{p}_{1}, \mathbf{p}_{2}\right\}, \\ \mathbf{h}_{j}=\frac{(-1)^{i}}{2} \Psi_{j} & \partial B_{i}, i=1,2,\end{cases}
$$

with the conditions

$$
\mathbf{h}_{j}(\mathbf{x})=C_{j}+\mathcal{O}\left(|\mathbf{x}|^{-1}\right), \quad \nabla \mathbf{h}_{j}(\mathbf{x})=\mathcal{O}\left(|\mathbf{x}|^{-2}\right), \quad p_{j}(\mathbf{x})=\mathcal{O}\left(|\mathbf{x}|^{-2}\right),
$$

for some constant $C_{j}$ as $|\mathbf{x}| \rightarrow \infty$.

In [2] singular functions $\left(\mathbf{h}_{j}, p_{j}\right)$ are constructed using the stream function formulation for which the bipolar coordinate system is used. The bipolar coordinates $(\zeta, \theta)$ are defined by

$$
\zeta=\log \frac{\sqrt{(x+a)^{2}+y^{2}}}{\sqrt{(x-a)^{2}+y^{2}}}, \quad \theta=\arg (x-a, y)-\arg (x+a, y)
$$


where $a$ is the number appeared in (2.3). It is worth mentioning that the singular function $q$ in (3.3) is nothing but $q=\zeta / 2 \pi$.

Suppose that $\partial B_{1}$ and $\partial B_{2}$ have the same radius $R$ for convenience. Let

$$
s=\sinh ^{-1}(a / R),
$$

and

$$
\mathbf{e}_{\zeta}=\frac{\nabla \zeta}{|\nabla \zeta|^{\prime}}, \quad \mathbf{e}_{\theta}=\frac{\nabla \theta}{|\nabla \theta|}
$$

Define two constants $A_{1}$ and $B_{1}$ by

$$
A_{1}:=\frac{1}{2 s-\tanh 2 s}, \quad B_{1}:=-\frac{1}{2 \cosh 2 s} A_{1} .
$$

Then, the velocity $\mathbf{h}_{1}$ is given by $\mathbf{h}_{1}=h_{1 \zeta} \mathbf{e}_{\zeta}+h_{1 \theta} \mathbf{e}_{\theta}$ where

$$
\begin{aligned}
& h_{1 \zeta}=\left(A_{1} \zeta+B_{1} \sinh 2 \zeta\right) \frac{1-\cosh \zeta \cos \theta}{\cosh \zeta-\cos \theta}, \\
& h_{1 \theta}=\sin \theta\left(A_{1}+2 B_{1} \cosh 2 \zeta-\frac{\sinh \zeta\left(A_{1} \zeta+B_{1} \sinh 2 \zeta\right)}{\cosh \zeta-\cos \theta}\right),
\end{aligned}
$$

and the pressure $p_{1}$ is given by

$$
p_{1}=\frac{2 \mu}{a}\left(\left(A_{1}-2 B_{1}\right) \cosh \zeta \cos \theta+B_{1} \cosh 2 \zeta \cos 2 \theta\right)-\frac{2 \mu}{a}\left(A_{1}-B_{1}\right) .
$$

The formulas for $\left(\mathbf{h}_{2}, p_{2}\right)$ are quite involved. But it is proved in [2] that

$$
\begin{aligned}
& \mathbf{h}_{2}=-A_{2} \zeta \mathbf{e}_{y}+A_{2} x(\nabla \zeta)^{\perp}+\mathbf{h}_{2 o}, \\
& p_{2}=-\frac{2 \mu}{a} A_{2} \sinh \zeta \sin \theta+p_{2 o},
\end{aligned}
$$

where $\left(\mathbf{h}_{20}, p_{20}\right)$ is a solution whose gradient is bounded regardless of $\epsilon$, and $A_{2}$ is the constant defined by

$$
A_{2}=-\frac{1}{2 s+\sinh 2 s} .
$$

It is proved in the same paper that if the background velocity field $\mathbf{U}$ is given by

$$
\mathbf{U}(x, y)=\left[\begin{array}{cc}
\alpha & \beta \\
\gamma & -\alpha
\end{array}\right]\left[\begin{array}{l}
x \\
y
\end{array}\right], \quad\left(\alpha^{2}+(\beta+\gamma)^{2} \neq 0\right)
$$

for some constants $\alpha, \beta$ and $\gamma$ and the background pressure $P=0$, and if $D_{1}$ and $D_{2}$ are disks of the same radius $R$ (so that $D_{j}=B_{j}$ for $j=1,2$ ), then the solution $(\mathbf{u}, p)$ admits a decomposition of the following form:

$$
(\mathbf{u}, p)=\alpha \frac{2}{\sqrt{R}} \epsilon^{3 / 2}\left(\mathbf{h}_{1}, p_{1}\right)+\frac{\beta+\gamma}{2} \sqrt{R \epsilon}\left(\mathbf{h}_{2}, p_{2}\right)+\left(\mathbf{u}_{0}, p_{0}\right),
$$


where $\left(\mathbf{u}_{0}, p_{0}\right)$ is a solution to the Stokes problem whose stress tensor is bounded. Thus we have

$$
\sigma[\mathbf{u}, p]=\alpha \frac{2}{\sqrt{R}} \epsilon^{3 / 2} \sigma\left[\mathbf{h}_{1}, p_{1}\right]+\frac{\beta+\gamma}{2} \sqrt{\operatorname{R} \epsilon} \sigma\left[\mathbf{h}_{2}, p_{2}\right]+\sigma\left[\mathbf{u}_{0}, p_{0}\right] .
$$

Since

$$
\left\|\sigma\left[\mathbf{h}_{1}, p_{1}\right]\right\|_{L^{\infty}\left(D^{e}\right)} \approx \epsilon^{-2} \text { and }\left\|\sigma\left[\mathbf{h}_{2}, p_{2}\right]\right\|_{L^{\infty}\left(D^{e}\right)} \approx \epsilon^{-1}
$$

as proved in [2], we have

$$
\|\sigma[\mathbf{u}, p]\|_{L^{\infty}\left(D^{e}\right)} \approx \epsilon^{-1 / 2},
$$

which says that the stress always blows up at the rate of $\epsilon^{-1 / 2}$ provided that $\mathbf{U}$ is linear as given in (5.13) and inclusions are circular. It is quite interesting and challenging to extend this result to the non-circular case.

\section{Acknowledgements}

This work is supported by NRF 2019R1A2B5B01069967 and 2020R1C1C1A01010882.

\section{References}

[1] H. Ammari, G. Ciraolo, H. Kang, H. Lee and K. Yun, Spectral analysis of the NeumannPoincaré operator and characterization of the stress concentration in anti-plane elasticity, Arch. Rational Mech. Anal., 208 (2013), 275-304.

[2] H. Ammari, H. Kang, D. W. Kim and S. Yu, Quantitative estimates for stress concentration of the Stokes flow between adjacent circular cylinders, arXiv:2003.06578.

[3] H. Ammari, H. Kang, H. Lee, J. Lee and M. Lim, Optimal bounds on the gradient of solutions to conductivity problems, J. Math. Pure. Appl., 88 (2007), 307-324.

[4] H. Ammari, H. Kang, H. Lee, M. Lim and H. Zribi, Decomposition theorems and fine estimates for electrical fields in the presence of closely located circular inclusions, J. Differ. Equations, 247 (2009), 2897-2912.

[5] H. Ammari, H. Kang and M. Lim, Gradient estimates for solutions to the conductivity problem, Math. Ann., 332(2) (2005), 277-286.

[6] I. Babuška, B. Andersson, P. Smith and K. Levin, Damage analysis of fiber composites. I. Statistical analysis on fiber scale, Comput. Methods Appl. Mech. Eng., 172 (1999), $27-77$.

[7] E. S. Bao, Y. Li and B. Yin, Gradient estimates for the perfect conductivity problem, Arch. Rational Mech. Anal., 193 (2009), 195-226.

[8] J. Bao, H. Li and Y. Li, Gradient estimates for solutions of the Lamé system with partially infinite coefficients, Arch. Rational Mech. Anal., 215 (2015), 307-351.

[9] E. S. Bao, Y. Li and B. Yin, Gradient estimates for the perfect and insulated conductivity problems with multiple inclusions, Commun. Partial Differential Equations, 35 (2010), 19822006.

[10] L. Berlyand and A. Kolpakov, Network approximation in the limit of small interparticle distance of the effective properties of a high-contrast random dispersed composite, Arch. Rational Mech. Anal., 159 (2001), 179-227. 
[11] E. Bonnetier, and M. Vogelius, An elliptic regularity result for a composite medium with touching fibers of circular cross-section, SIAM J. Math. Anal., 31 (2000), 651-677.

[12] H. Dong and H. Li, Optimal estimates for the conductivity problem by Green's function method, Arch. Ration. Mech. Anal., 231 (2019), 1427-1453.

[13] Y. Gorb, Singular behavior of electric field of high contrast concentrated composites, SIAM Multiscale Model. Simul., 13 (2015), 1312-1326.

[14] Y. Gorb and A. Novikov, Blow-up of solutions to a $p$-Laplace equation, SIAM Multiscale Model. Simul., 10 (2012), 727-743.

[15] P. Grisvard, Boundary Value Problems in Non-Smooth Domains, Pitman, London, 1985.

[16] H. Kang, H. Lee and K. Yun, Optimal estimates and asymptotics for the stress concentration between closely located stiff inclusions, Math. Ann., 363 (2015), 1281-1306.

[17] H. Kang, M. Lim and K. Yun, Asymptotics and computation of the solution to the conductivity equation in the presence of adjacent inclusions with extreme conductivities, J. Math. Pure. Appl., 99 (2013), 234-249.

[18] H. Kang, M. Lim and K. Yun, Characterization of the electric field concentration between two adjacent spherical perfect conductors, SIAM J. Appl. Math., 74 (2014), 125-146.

[19] H. Kang and S. Yu, Quantitative characterization of stress concentration in the presence of closely spaced hard inclusions in two-dimensional linear elasticity, Arch. Rati. Mech. Anal., 232 (2019), 121-196.

[20] H. Kang and S. Yu, A proof of the Flaherty-Keller formula on the effective property of densely packed elastic composites, Cal. Vari. PDE, 59 (2020), 22.

[21] H. Kang and K. Yun, Optimal estimates of the field enhancement in presence of a bow-tie structure of perfectly conducting inclusions in two dimensions, J. Differential Equations, 266 (2019), 5064-5094.

[22] H. Kang and K. Yun, Precise estimates of the field excited by an emitter in presence of closely located inclusions of a bow-tie shape, J. Math. Anal. Appl., 479(2) (2019), 1670-1707.

[23] H. Kang and K. Yun, Quantitative estimates of the field excited by an emitter in a narrow region between two circular inclusions, Q. Appl. Math. LXXVII, 4 (2019), 861-873.

[24] H. Kang and K. Yun, Quantitative estimates for enhancement of the field excited by an emitter due to presence of two closely located spherical inclusions, J. Differential Equations, 269 (2020), 2977-3002.

[25] V. A. Kondratiev, Boundary-value problems for elliptic equations in domains with conical or angular points, Trans. Moscow Math. Soc., 16 (1967), 227-313.

[26] V. A. Kozlov, V. G. Maz'ya and J. Rossmann, Elliptic Boundary Value Problems in Domains with Point Singularities, Amer. Math. Soc., Mathematical Surveys and Monographs, vol. 52, Providence, RI, 1997.

[27] J. Lekner, Electrostatics of two charged conducting spheres, Proc. R. Soc. A, 468 (2012), 28292848.

[28] H. Li, Y. Li, E. S. Bao and B. Yin, Derivative estimates of solutions of elliptic systems in narrow domains, Q. Appl. Math., 72 (2014), 589-596.

[29] H. Li, F. Wang and L. Xu, Characterization of electric fields between two spherical perfect conductors with general radii in 3D, J. Differential Equations, 267 (2019), 6644-6690.

[30] Y. Li and L. Nirenberg, Estimates for elliptic system from composite material, Commun. Pure Appl. Math., LVI (2003), 892-925.

[31] Y. Li and M. Vogelius, Gradient estimates for solution to divergence form elliptic equation with discontinuous coefficients, Arch. Ration. Mech. Anal., 153 (2000), 91-151.

[32] M. Lim and S. Yu, Asymptotics of the solution to the conductivity equation in the presence 
of adjacent circular inclusions with finite conductivities, J. Math. Anal. Appl., 421 (2015), 131-156.

[33] M. Lim and K. Yun, Blow-up of electric fields between closely spaced spherical perfect conductors, Commun. Partial Differential Equations, 34 (2009), 1287-1315.

[34] M. Lim and K. Yun, Strong influence of a small fiber on shear stress in fiber-reinforced composites, J. Differential Equations, 250 (2011), 2402-2439.

[35] S. Yu and H. Ammari, Plasmonic interaction between nanosphere, SIAM Rev., 60 (2018), 356-385.

[36] S. Yu and H. Ammari, Hybridization of singular plasmons via transformation optics, Proceedings of the National Academy of Sciences, 116 (2019), 13785-13790.

[37] K. Yun, Estimates for electric fields blown up between closely adjacent conductors with arbitrary shape, SIAM J. Appl. Math., 67 (2007), 714-730.

[38] K. Yun, Optimal bound on high stresses occurring between stiff fibers with arbitrary shaped cross sections, J. Math. Anal. Appl., 350 (2009), 306-312.

[39] K. Yun, An optimal estimate for electric fields on the shortest line segment between two spherical insulators in three dimensions, J. Differential Equations, 261 (2016), 148-188. 